Review

\title{
The Association between Bone Turnover Markers and Fracture in People with Diabetes: A Systematic Review and Meta-Analysis
}

\author{
Katrine Hygum ${ }^{1,2,+, *}$, Jakob Starup-Linde ${ }^{1,+}$, Torben Harsl $\varnothing f^{1}$, Bente Langdahl ${ }^{1}$
}

1. Department of Endocrinology and Internal Medicine, Aarhus University Hospital, Palle JuulJensens Boulevard 99, 8200 Aarhus N, Denmark; E-Mails: katrhygu@rm.dk; Jakob.Linde@auh.rm.dk; torbhars@rm.dk; bente.langdahl@aarhus.rm.dk

2. Department of Medicine, Regional Hospital West Jutland, Herning, Gl. Landevej 61, 7400 Herning, Denmark

$\dagger$ These authors contributed equally to this work.

* Correspondence: Katrine Hygum; E-Mail: katrhygu@rm.dk

Academic Editor: P. Hemachandra Reddy

Special Issue: Diabetes in the Elderly

\section{OBM Geriatrics}

2019, volume 3 , issue 4

doi:10.21926/obm.geriatr.1904090
Received: August 29, 2019

Accepted: November 18, 2019

Published: December 04, 2019

\begin{abstract}
Background: The increased risk of fractures in individuals with diabetes has been underestimated by conventional fracture predictors such as the bone mineral density Tscore or the Fracture Risk Assessment Tool. The present study conducted a systematic review and a meta-analysis to investigate the association between the levels of bone turnover markers (BTMs) and fractures in patients with diabetes.
\end{abstract}

Methods: We conducted a systematic literature search. Eligibility criteria were studies investigating BTMs in patients with diabetes with/without fractures. For the meta-analysis, we primarily used the fixed effects model. The estimates were reported as the standardized mean difference (SMD).

Results: We included eight observational studies. The levels of osteocalcin, procollagen type 1 amino terminal propeptide (P1NP), and insulin-like growth factor-1 (IGF-1) were found to

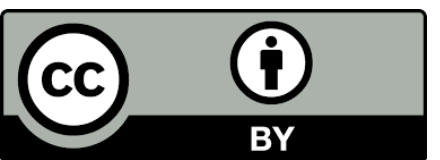

(C) 2019 by the author. This is an open access article distributed under the conditions of the Creative Commons by Attribution License, which permits unrestricted use, distribution, and reproduction in any medium or format, provided the original work is correctly cited. 
be significantly lower in individuals with fracture than in those without fracture $(-0.36[-0.46$, $-0.26]$ ) (SMD [95\% confidence interval], $(-0.57[-0.75,-0.40])$, and $(-0.50[-0.61,-0.39])$, respectively. The levels of $\mathrm{N}$-terminal cross-linked telopeptide of type 1 collagen (NTX), sclerostin, and bone-specific alkaline phosphatase (BAP) were significantly higher in patients with fracture $(0.24[0.13,0.35],(0.47[0.29,0.65])$, and $(0.14[0.01,0.27])$, respectively, whereas C-terminal cross-linked telopeptide (CTX) showed no difference between the groups. The sensitivity analysis revealed an effect of study size but not study design.

Conclusions: The results suggest that BTMs could be associated with fracture status in individuals with diabetes with lower levels of formative BTMs and IGF-1 and higher levels of NTX, sclerostin, and BAP in these individuals than in those without fracture. However, some results depended on one large study.

\section{Keywords}

Diabetes mellitus; bone remodeling; fractures; bone

\section{Introduction}

Diabetes mellitus type 1 (T1D) and type 2 (T2D) are diseases prevalent among the geriatric population with both associated with increased susceptibility to fractures [1]. For example, hip and vertebral fractures have been reported to be associated with increased morbidity and mortality $[2,3]$. Briefly, the fall risk may be increased in individuals with diabetes owing to retinopathy, neuropathy, and hypoglycemia; however several studies have demonstrated that the increased risk of fractures in individuals with diabetes persisted after adjustment for falls [4]. Rather, cellular and molecular changes, including incorporation of advanced glycation end products (AGEs) in the bone collagen matrix [5], increased levels of sclerostin [6], a direct negative effect of hyperglycemia [7], and possible harmful side-effects of anti-diabetic medication [8] may lead to fragile bones in patients with diabetes, as reviewed by Napoli on behalf of the International Osteoporosis Foundation Bone and Diabetes Working Group [9]. Furthermore, the bone turnover process in itself could be impaired. A meta-analysis has shown that circulating levels of bone turnover markers (BTMs) are significantly lower in individuals with T1D and T2D compared with those without diabetes [10].

Fracture risk is traditionally evaluated by bone mineral density (BMD) measured by dual-energy X-ray absorptiometry (DXA) [11] or by the Fracture Risk Assessment Tool (FRAX) [12]. For T1D and T2D, both BMD and FRAX underestimate the risk of fracture $[13,14]$, thus warranting additional fracture predictors in these populations. Circulating BTMs reflect the bone turnover process in terms of bone formation and bone resorption. BTMs are widely used to monitor osteoporosis treatment, and several studies have indicated that BTMs may predict the fracture risk in populations without diabetes $[15,16]$. The levels of BTMs are generally reduced in individuals with diabetes; however, it is unknown whether levels of BTMs in diabetes may predict fracture risk.

The present study conducted a systematic review and a meta-analysis evaluating and comparing the levels of BTMs and bone signaling markers in patients with diabetes with and without fractures. 


\section{Materials and Methods}

We used the Preferred Reporting Items for Systematic Reviews and Meta-Analyses (PRISMA) guidelines [17].

\subsection{Literature Search}

We performed a systematic literature search on January 2, 2017, and updated this on August 6, 2018. We studied the databases PubMed (1966-2018) and Embase (1974-2018) using free-text search terms "Fracture" and "diabetes" in combination with the following free-text search terms: "BALP," "BAP," "alkaline phosphatase," "bone alkaline phosphatase," "RANKL," "sclerostin," "Nterminal telopeptide," "NTX," "opg," "osteoprotegerin," "osteocalcin," "bone GLA protein," "procollagen type 1," "PINP," "P1NP," "type 1 collagen," "CTX," and "bone turnover." We used the free-text search terms to avoid omitting any relevant literature. No limitations were applied during the literature search (e.g., publication date and language).

\subsection{Eligibility Criteria}

The eligibility criteria were studies investigating the levels of bone formation markers (procollagen type 1 amino terminal propeptide [P1NP], osteocalcin, and bone-specific alkaline phosphatase [BAP]), levels of bone resorption markers (C-terminal cross-linked telopeptide [CTX], $\mathrm{N}$-terminal cross-linked telopeptide of type 1 collagen [NTX], and tartrate-resistant acid phosphatase [TRAP]), and levels of bone turnover signaling markers (osteoprotegerin [OPG], receptor activator of nuclear factor kappa-B ligand [RANKL], and sclerostin) in patients with diabetes with and without fractures. We included both prevalent fractures and incident fractures and excluded duplicate studies. Furthermore, we excluded studies reporting data from identical study populations and hence excluded articles by Kanazawa et al. [18-22], Notsu et al. [23], Miyake et al. [24], Tanaka et al. [25], and Yamamoto et al. [26, 27] as all participants from these studies appeared to be included in the most recent study by Kanazawa et al. in 2018 [28]. Similarly, for another study by the same author [29], we included only the results from analyses of parathyroid hormone (PTH) and 1,25 Vitamin D, as all other analyses were performed in the study published by Kanazawa et al. in 2018 [28].

\subsection{Study Characteristics}

Two authors (KH and JS-L) screened all articles that were retrieved from the literature search by title and abstract, and subsequently read all studies that were possibly eligible for inclusion. We collected data on the levels of BTMs for each study, and if possible, we used subgroups (e.g., for gender [men/women] or diabetes type [T1D/T2D]). If several publications originated from the same author in the same year, we denoted each publication by author name followed by a letter ( $a, b$, etc.). We did not contact authors concerning additional study data; however, we provided further data from our own study [30]. We collected information on potential confounders such as participant characteristics (age and body mass index), levels of glycated hemoglobin (HbA1c) and plasma glucose, and blood sampling conditions. We evaluated funnel plots visually to assess the risk of publication bias. Two authors (KH and JS-L) independently evaluated the quality of the 
studies according to the modified Newcastle-Ottawa Scale (NOS) adapted for cross-sectional studies [31].

\subsection{Statistics}

We captured the mean values and standard deviations (SDs) or 95\% confidence intervals (Cls) of biochemical markers from the included studies. We implemented pooled analyses if at least three populations were available. We analyzed the standardized mean difference (SMD), which is the mean outcome between the groups divided by the SD of the outcome among the participants. The SMD considers assay and inter-laboratory differences as percentages are calculated. In addition, we calculated the common weighted mean difference (MD). If the SMD and MD reported the same effect, we reported only the SMD in the results section of the article. For analysis, we primarily used the fixed effects model and secondarily the random-effects model. The fixed-effects model assumes that all studies report on the same effect size and larger studies weigh more than smaller studies in the analyses. Contrary to this, the random effects model assumes that the effect size is different in the included studies owing to heterogeneity or different study characteristics and weighs large and small studies accordingly. Furthermore, we performed sensitivity analyses excluding specific studies to evaluate whether the observed findings could be attributed to the effects of study size or design. We determined the heterogeneity among studies by $I^{2}$ analysis and performed subgroup analysis in individuals with T2D. For all analyses, we used the RevMan 5.3 software program.

\section{Results}

\subsection{Study Selection}

The literature search recovered 3,246 articles. After the removal of duplicate and screening of abstracts and full-texts, we included eight studies (Figure 1). Seven studies had a cross-sectional design, whereas one study was a cohort study. All studies were published articles. Most studies reported on at least four different BTMs, except for two studies with data on only three and two BTMs, respectively. See Table 1 for study details. One study reported on patients with T1D, another reported on both T1D and T2D, and a third study reported on unspecified diabetes, whereas all other studies investigated patients with T2D $(n=5)$. If possible, results were stratified by gender. The number of participants ranged from 20 in the smallest study to approximately 1,000 in the largest. The number of studies evaluating each BTM varied greatly from three studies reporting levels of P1NP to seven studies describing the levels of osteocalcin. The included studies allowed for investigation of the bone turnover and bone signaling markers, such as total osteocalcin, P1NP, BAP, CTX, NTX, sclerostin, insulin-like growth factor-1 (IGF-1), PTH, HbA1c, and Vitamin D. We included studies with results concerning $\mathrm{HbA} 1 \mathrm{c}$, as previous data suggested an association between fracture status and levels of HbA1c [32-34]; however, results are conflicting for patients with T2D. Furthermore, we included studies that investigated the levels of Vitamin D, as this is an important regulator of calcium homeostasis and bone turnover. All but one study excluded participants with renal disease, an important possible confounder. Most studies excluded participants taking drugs that could affect bone metabolism (including specific antiosteoporotic therapy) ( $n=7)$; however, one study did not provide any information regarding this. 
Concerning vitamin D supplementation, three studies excluded participants taking vitamin D supplementation, two studies included these participants, and three studies did not provide any information. Almost all studies included only non-traumatic fractures [28-30, 35-38], whereas one study included both non-traumatic and traumatic fractures [34]. For information on different fracture sites, see Table 1 . Concerning the time period between fracture incidence and biochemical measurements, one study reported that only participants with fractures that had occurred more than 5 months ago were included [37], whereas seven other studies did not specify this. Three studies were from Asia [28, 29, 36], three from Europe [30, 34, 38], one from North America [37], and one from the Middle East [35]. The quality of the study was generally high, with NOS ranging from 6 to 10. The heterogeneity ranged between 0\% (BAP) and 94\% (osteocalcin). Based on funnel plots, we did not suspect any publication bias.

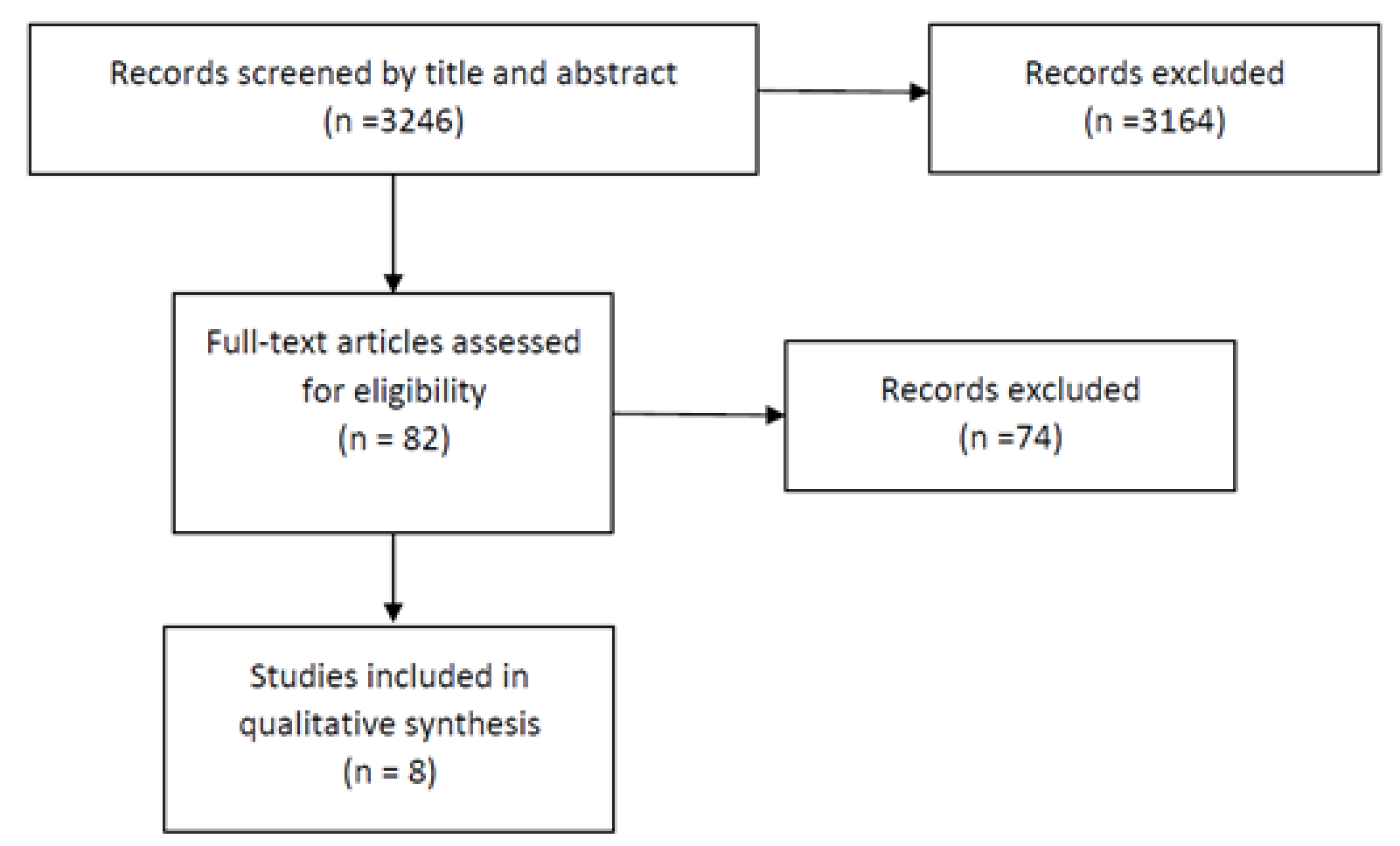

Figure 1 Flow diagram of the studies. 
Table 1 Characteristics of the included studies.

\begin{tabular}{|c|c|c|c|c|c|c|c|c|c|c|c|c|}
\hline Study & Participants & Source & $\begin{array}{l}\text { Age } \\
\text { (years) }\end{array}$ & $\begin{array}{l}\text { BMI } \\
\left(\mathrm{kg} / \mathrm{m}^{2}\right)\end{array}$ & $\begin{array}{l}\text { Additional } \\
\text { information } \\
\text { including } \\
\text { fracture site }\end{array}$ & $\begin{array}{l}\text { Anti- } \\
\text { osteoporotic } \\
\text { medication }\end{array}$ & HbA1c (\%) & $\begin{array}{l}\text { P-Glucose } \\
\text { (mg/dL) }\end{array}$ & BTM & $\begin{array}{l}\text { Fasting } \\
\text { samples }\end{array}$ & $\begin{array}{l}\text { Renal } \\
\text { Disease }\end{array}$ & $\begin{array}{l}\text { NOS } \\
\text { quality } \\
\text { score }\end{array}$ \\
\hline \multicolumn{13}{|l|}{$\begin{array}{l}\text { Case- } \\
\text { control }\end{array}$} \\
\hline $\begin{array}{l}\text { Dai et al. } \\
(2016)[36]\end{array}$ & $\begin{array}{l}15 \text { cases, } 5 \\
\text { controls }\end{array}$ & $\begin{array}{l}\text { Singapore } \\
\text { Chinese } \\
\text { Health } \\
\text { Study } \\
\text { (population } \\
\text { based) }\end{array}$ & $45-74$ & - & $\begin{array}{l}\text { Subgroup in a } \\
\text { larger study. } \\
\text { Incident hip } \\
\text { fractures. }\end{array}$ & $\begin{array}{l}\text { No } \\
\text { information }\end{array}$ & - & - & $\begin{array}{l}\text { BAP, OC, } \\
\text { P1NP, } \\
\text { CTX, NTX }\end{array}$ & no & $\begin{array}{l}\text { No } \\
\text { informati } \\
\text { on }\end{array}$ & 6 \\
\hline \multicolumn{13}{|l|}{$\begin{array}{l}\text { Cross- } \\
\text { sectional }\end{array}$} \\
\hline $\begin{array}{l}\text { Kanazawa } \\
\text { et al. } \\
\text { (2009)a[2 } \\
\text { 9] }\end{array}$ & $\begin{array}{l}76 \text { cases, } \\
172 \\
\text { controls }\end{array}$ & $\begin{array}{l}\text { Shimane } \\
\text { University } \\
\text { Hospital } \\
\text { (hospital } \\
\text { based) }\end{array}$ & $\begin{array}{l}62.5 \\
\text { (cases), } \\
57.5 \\
\text { (controls) }\end{array}$ & $\begin{array}{l}23.0 \\
\text { (cases), } \\
23.8 \\
\text { (controls) }\end{array}$ & $\begin{array}{l}\text { T2D, men. } \\
\text { Prevalent } \\
\text { vertebral } \\
\text { fractures. }\end{array}$ & $\begin{array}{l}\text { Excluded } \\
\text { drugs } \\
\text { affecting } \\
\text { bone } \\
\text { metabolism } \\
\text { and vitamin D }\end{array}$ & $\begin{array}{l}9.0 \text { (cases), } \\
9.1 \\
\text { (controls) }\end{array}$ & $\begin{array}{l}169 \\
\text { (cases), } \\
172 \\
\text { (controls) }\end{array}$ & $\begin{array}{l}\text { a IGF-1, } \\
\text { PTH, } 25 \\
\text { OHD, BAP, } \\
\text { OC, NTX }\end{array}$ & yes & $\begin{array}{l}\text { Renal } \\
\text { disease } \\
\text { excluded }\end{array}$ & 7 \\
\hline $\begin{array}{l}\text { Kanazawa } \\
\text { et al. } \\
(2018)[28]\end{array}$ & $\begin{array}{l}\text { Men: } 219 \\
\text { cases, } 363 \\
\text { controls. } \\
\text { Women: } \\
145 \text { cases, } \\
267\end{array}$ & $\begin{array}{l}\text { Shimane } \\
\text { University } \\
\text { Hospital } \\
\text { (hospital } \\
\text { based) }\end{array}$ & $\begin{array}{l}\text { Men: } 68.0 \\
\text { (cases), } \\
64.7 \\
\text { (controls) } \\
\text { Women: } \\
70.9\end{array}$ & $\begin{array}{l}\text { Men: } 23.5 \\
\text { (cases), } \\
23.4 \\
\text { (controls) } \\
\text { Women: } \\
24.3\end{array}$ & $\begin{array}{l}\text { T2D. All } \\
\text { women } \\
\text { postmenopau } \\
\text { sal. Prevalent } \\
\text { vertebral } \\
\text { fractures. }\end{array}$ & $\begin{array}{l}\text { Excluded } \\
\text { drugs } \\
\text { affecting } \\
\text { bone } \\
\text { metabolism, } \\
\text { no }\end{array}$ & $\begin{array}{l}\text { Men: 9.0, } \\
\text { (cases), } 9.3 \\
\text { (controls) } \\
\text { Women: } 8.9 \\
\text { (cases), } 9.0 \\
\text { (controls) }\end{array}$ & - & $\begin{array}{l}\text { OC, BAP, } \\
\text { u-NTX, } \\
\text { IGF-1 }\end{array}$ & yes & $\begin{array}{l}\text { No } \\
\text { informati } \\
\text { on, but } \\
\text { creatinin } \\
\text { e in } \\
\text { normal }\end{array}$ & 8 \\
\hline
\end{tabular}


OBM Geriatrics 2019; 3(4), doi:10.21926/obm.geriatr.1904090

\begin{tabular}{|c|c|c|c|c|c|c|c|c|c|c|c|c|}
\hline & controls & & $\begin{array}{l}\text { (cases), } \\
65.9 \\
\text { (controls) }\end{array}$ & $\begin{array}{l}\text { (cases), } \\
24.7 \\
\text { (controls) }\end{array}$ & & $\begin{array}{l}\text { information } \\
\text { on vitamin } D\end{array}$ & & & & & range. & \\
\hline $\begin{array}{l}\text { Neumann } \\
\text { et al. } \\
(2014)[34]\end{array}$ & $\begin{array}{l}104 \\
\text { controls, } 24 \\
\text { cases. }\end{array}$ & $\begin{array}{l}\text { Jena } \\
\text { University } \\
\text { Hospital } \\
\text { (hospital } \\
\text { based) }\end{array}$ & $\begin{array}{l}46 \text { (cases), } \\
42.7 \\
\text { (controls) }\end{array}$ & $\begin{array}{l}26.1 \\
\text { (cases), } \\
26.9 \\
\text { (controls) }\end{array}$ & $\begin{array}{l}\text { T1D } 128 \\
\text { participants. } \\
\text { Prevalent } \\
\text { fractures of } \\
\text { all types. }\end{array}$ & $\begin{array}{l}\text { Excluded } \\
\text { drugs } \\
\text { affecting } \\
\text { bone } \\
\text { metabolism, } \\
\text { no } \\
\text { information } \\
\text { on vitamin D }\end{array}$ & $\begin{array}{l}8.2 \text { (cases), } \\
7.6 \\
\text { (controls) } \\
\mathrm{mmol} / \mathrm{l}\end{array}$ & - & CTX, OC & yes & $\begin{array}{l}\text { eGFR }<30 \\
\mathrm{~mL} / \mathrm{min} \\
\text { excluded }\end{array}$ & 8 \\
\hline $\begin{array}{l}\text { Starup- } \\
\text { Linde et } \\
\text { al. } \\
(2016)[30]\end{array}$ & $\begin{array}{l}\text { T1D: } 21 \\
\text { (cases), } 80 \\
\text { (controls). } \\
\text { T2D: } 13 \\
\text { (cases), } 86 \\
\text { (controls). }\end{array}$ & $\begin{array}{l}\text { Aarhus \& } \\
\text { Aalborg } \\
\text { University } \\
\text { Hospitals } \\
\text { (hospital } \\
\text { based) }\end{array}$ & $\begin{array}{l}\text { T1D: } 62 \\
\text { (cases), } 60 \\
\text { (controls). } \\
\text { T2D: } 66 \\
\text { (cases), } 65 \\
\text { (controls). }\end{array}$ & $\begin{array}{l}\text { T1D: } 25 \\
\text { (cases), } 26 \\
\text { (controls). } \\
\text { T2D: } 31 \\
\text { (cases), } 31 \\
\text { (controls). }\end{array}$ & $\begin{array}{l}\text { T2D } 96 \\
\text { participants, } \\
\text { T1D } 101 \\
\text { participants. } \\
\text { Prevalent } \\
\text { vertebral } \\
\text { fractures and } \\
\text { incident } \\
\text { fractures. }\end{array}$ & $\begin{array}{l}\text { Excluded } \\
\text { drugs } \\
\text { affecting } \\
\text { bone } \\
\text { metabolism, } \\
\text { vitamin D } \\
\text { supplementat } \\
\text { ion allowed }\end{array}$ & $\begin{array}{l}\text { T1D: } 7.9 \\
\text { (cases), } 8.1 \\
\text { (controls). } \\
\text { T2D: } 7.9 \\
\text { (cases), } 8.0 \\
\text { (controls). }\end{array}$ & $\begin{array}{l}\text { T1D: } 175 \\
\text { (cases), } \\
196 \\
\text { (controls). } \\
\text { T2D: } 198 \\
\text { (cases), } \\
196 \\
\text { (controls). }\end{array}$ & $\begin{array}{l}\text { CTX, } \\
\text { P1NP, OC, } \\
\text { Sclerostin, } \\
25 \text { OHD }\end{array}$ & no & $\begin{array}{l}\text { Patients } \\
\text { with } \\
\text { renal } \\
\text { disease } \\
\text { excluded. }\end{array}$ & 10 \\
\hline $\begin{array}{l}\text { Zhukouska } \\
\text { ya et al. } \\
(2014)[38]\end{array}$ & $\begin{array}{l}34 \text { cases, } 65 \\
\text { controls }\end{array}$ & $\begin{array}{l}\text { Outpatient } \\
\text { clinic } \\
\text { (hospital } \\
\text { based) }\end{array}$ & $\begin{array}{l}66.4 \\
\text { (cases), } \\
65.4 \\
\text { (controls) }\end{array}$ & $\begin{array}{l}28.9 \\
\text { (cases), } \\
29.8 \\
\text { (controls) }\end{array}$ & $\begin{array}{l}\text { T2D, } 99 \\
\text { postmenopau } \\
\text { sal women. } \\
\text { Prevalent } \\
\text { vertebral } \\
\text { fractures. }\end{array}$ & $\begin{array}{l}\text { Excluded } \\
\text { drugs } \\
\text { affecting } \\
\text { bone } \\
\text { metabolism, } \\
\text { vitamin D } \\
\text { supplementat } \\
\text { ion allowed }\end{array}$ & $\begin{array}{l}6.9 \text { (cases), } \\
6.6 \\
\text { (controls) }\end{array}$ & - & $\begin{array}{l}\text { CTX, OC, } \\
25 \mathrm{OHD}\end{array}$ & Yes & $\begin{array}{l}\text { Chronic } \\
\text { renal } \\
\text { failure } \\
\text { excluded }\end{array}$ & 8 \\
\hline
\end{tabular}


OBM Geriatrics 2019; 3(4), doi:10.21926/obm.geriatr.1904090

\begin{tabular}{|c|c|c|c|c|c|c|c|c|c|c|c|c|}
\hline $\begin{array}{l}\text { Ardawi et } \\
\text { al. } \\
2013[35]\end{array}$ & $\begin{array}{l}118 \text { cases, } \\
364 \\
\text { controls }\end{array}$ & $\begin{array}{l}\text { King } \\
\text { Abdulaziz } \\
\text { University } \\
\text { Hospital } \\
\text { (hospital } \\
\text { based) }\end{array}$ & $\begin{array}{l}65.5 \\
\text { (cases), } \\
57.7 \\
\text { (controls) }\end{array}$ & $\begin{array}{l}33.4 \\
\text { (cases), } \\
33.4 \\
\text { (controls) }\end{array}$ & $\begin{array}{l}\text { T2D, } \\
\text { postmenopau } \\
\text { sal women. } \\
\text { Prevalent } \\
\text { vertebral } \\
\text { fractures. }\end{array}$ & $\begin{array}{l}\text { Excluded } \\
\text { drugs } \\
\text { affecting } \\
\text { bone } \\
\text { metabolism } \\
\text { and vitamin D }\end{array}$ & $\begin{array}{l}10.5 \% \\
\text { (cases), } \\
9.6 \% \\
\text { (controls) }\end{array}$ & $\begin{array}{l}149.8 \\
\text { (cases), } \\
157.3 \\
\text { (controls) }\end{array}$ & $\begin{array}{l}\text { PTH, IGF- } \\
1,25 \\
\text { OHD, } \\
\text { sclerostin, } \\
\text { OC, P1NP, } \\
\text { CTX, u- } \\
\text { NTX }\end{array}$ & Yes & $\begin{array}{l}\text { Renal } \\
\text { bone } \\
\text { disease } \\
\text { excluded }\end{array}$ & 9 \\
\hline $\begin{array}{l}\text { Heilmeier } \\
\text { et al. } \\
2015[37]\end{array}$ & $\begin{array}{l}19 \text { cases, } 20 \\
\text { controls }\end{array}$ & $\begin{array}{l}\text { Diabetic } \\
\text { and } \\
\text { orthopedic } \\
\text { clinics }\end{array}$ & $\begin{array}{l}63.3 \\
\text { (cases), } \\
59.6 \\
\text { (controls) }\end{array}$ & $\begin{array}{l}28.9 \\
\text { (cases), } \\
27.8 \\
\text { (controls) }\end{array}$ & $\begin{array}{l}\text { T2D. Women } \\
\text { age } 50-75 . \\
\text { Prevalent } \\
\text { fragility } \\
\text { fractures. }\end{array}$ & $\begin{array}{l}\text { Excluded } \\
\text { drugs } \\
\text { affecting } \\
\text { bone } \\
\text { metabolism } \\
\text { and vitamin D }\end{array}$ & $\begin{array}{l}8.0 \% \\
\text { (cases), } \\
7.9 \% \\
\text { (controls) }\end{array}$ & $\begin{array}{l}151.0 \\
\text { (cases), } \\
159.6 \\
\text { (controls) }\end{array}$ & $\begin{array}{l}25 \text { OHD, } \\
\text { PTH, Ca, } \\
\text { Sclerostin, } \\
\text { CTX, P1NP }\end{array}$ & Yes & $\begin{array}{l}\text { Renal } \\
\text { disease } \\
\text { excluded }\end{array}$ & 9 \\
\hline
\end{tabular}

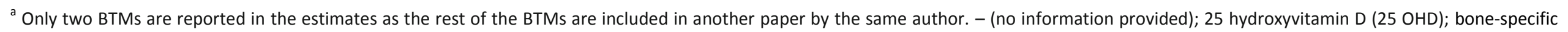

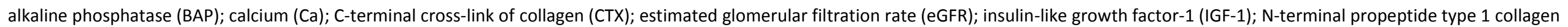

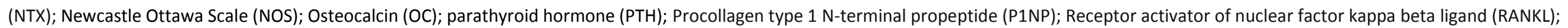
Tartrate resistant acid phosphatase (TRAP); type 2 diabetes (T2D); type 1 diabetes (T1D); undercarboxylated osteocalcin (ucOC); urinary NTX (U-NTX). 


\subsection{Results of Bone Turnover Markers in Primary Analyses: The Fixed Effects Model}

For formative BTMs, the levels of osteocalcin (SMD $=-0.36[95 \% \mathrm{Cl}:-0.46,-0.26]$ ) and P1NP $(S M D=-0.57(95 \% \mathrm{Cl}:-0.75,-0.40)$ were significantly lower in patients with diabetes with fracture as compared with those without fracture (Figures 2 and 3, respectively). The levels of the mineralization marker, BAP, were significantly higher in patients with diabetes and fracture compared with those without fracture (SMD $=0.14$ [95\% Cl: 0.01, 0.27]; Figure 4). For resorptive BTMs, only NTX levels were significantly higher in patients with diabetes and fracture compared with those without fracture (SMD $=0.24[95 \% \mathrm{Cl}: 0.13,0.35]$; Figure 5). For bone signaling markers, the levels of sclerostin were significantly higher in patients with diabetes and fracture as compared with those without fracture (SMD $=0.47$ [95\% Cl: 0.29, 0.65]; Figure 6a). Moreover, similar results were obtained when limiting the analyses to participants with T2D (SMD $=0.62$ [95\% Cl: 0.43, 0.81]; Figure 6b). The IGF-1-levels were significantly lower in individuals with diabetes and fracture compared with those without fracture (SMD $=-0.50(95 \% \mathrm{Cl}:-0.61,-0.39)$; Figure 7). The levels of Vitamin $D$ were borderline higher in patients with diabetes with fracture compared with those without fracture (SMD $=0.13$ (95\% Cl: $-0.01,0.27$ ], Figure 8). For osteocalcin, P1NP, BAP, NTX, sclerostin, IGF-1, and vitamin D, the results and the significance of the results did not change when applying the MD. For CTX, there was no difference between the groups when applying the SMD; however, when using the MD, the levels of CTX were significantly lower in patients with diabetes with fracture as compared with those without fracture $(M D=-0.03 \mathrm{ng} / \mathrm{mL}$ (95\% Cl: $-0.05,-0.01]$ ). For PTH and HbA1c, all results were non-significant. Furthermore, the results did not change when the analyses were limited to participants with T2D.

\begin{tabular}{|c|c|c|c|c|c|c|c|c|c|c|c|}
\hline \multirow[b]{2}{*}{ Study or Subgroup } & \multicolumn{3}{|c|}{ Fracture } & \multicolumn{3}{|c|}{ No fracture } & \multirow[b]{2}{*}{ Weight } & Std. Mean Difference & \multirow{2}{*}{\multicolumn{3}{|c|}{$\begin{array}{l}\text { Std. Mean Difference } \\
\text { IV, Fixed, } 95 \% \mathrm{Cl}\end{array}$}} \\
\hline & Mean & SD & Total & Mean & SD & Total & & IV, Fixed, $95 \% \mathrm{CI}$ & & & \\
\hline Ardawi et al. 2013b & 8.01 & 2.29 & 118 & 15.56 & 5.52 & 364 & $19.4 \%$ & $-1.53[-1.76,-1.30]$ & $\longrightarrow$ & & \\
\hline Dai et al. 2016 & 14 & 5.6 & 15 & 12.62 & 5.6 & 5 & $1.0 \%$ & $0.24[-0.78,1.25]$ & & & \\
\hline Kanazawa 2018 men & 5.3 & 3.1 & 219 & 5.5 & 3.3 & 363 & $36.2 \%$ & $-0.06[-0.23,0.11]$ & $\rightarrow-$ & - & \\
\hline Kanazawa 2018 women & 6.7 & 3.5 & 145 & 7.2 & 3.7 & 267 & $24.9 \%$ & $-0.14[-0.34,0.06]$ & $\rightarrow$ & & \\
\hline Neumann et al. 2014 & 16 & 6.8 & 24 & 15.6 & 7.5 & 104 & $5.2 \%$ & $0.05[-0.39,0.50]$ & & & \\
\hline Starup-Linde et al. 2016 T1D & 20.1 & 6.24 & 21 & 19.38 & 6.02 & 80 & $4.4 \%$ & $0.12[-0.36,0.60]$ & & & \\
\hline Starup-Linde et al. 2016 T2D & 14.06 & 5.34 & 13 & 15.74 & 6.21 & 82 & $3.0 \%$ & $-0.27[-0.86,0.31]$ & & & \\
\hline Zhukouskaya et al. 2014 & 4.6 & 3.4 & 34 & 5.2 & 3.1 & 65 & $5.9 \%$ & $-0.19[-0.60,0.23]$ & & & \\
\hline Total $(95 \% \mathrm{Cl})$ & & & 589 & & & 1330 & $100.0 \%$ & $-0.36[-0.46,-0.26]$ & & & \\
\hline \multicolumn{9}{|c|}{$\begin{array}{l}\text { Heterogeneity: } \mathrm{Chi}^{2}=125.91, \mathrm{df}=7(\mathrm{P}<0.00001) ;\left.\right|^{2}=94 \% \\
\text { Test for overall effect: } Z=7.03(P<0.00001)\end{array}$} & $\begin{array}{ccc}-1 & -0.5 & 0 \\
\text { Lower with fracture }\end{array}$ & $\begin{array}{l}0.5 \\
\text { Higher with }\end{array}$ & $\begin{array}{l}1 \\
\text { fracti }\end{array}$ \\
\hline
\end{tabular}

Figure 2 Pooled analysis of osteocalcin levels in persons with diabetes with fracture compared with those with diabetes without fracture. Studies with several populations are explained as the author name followed by a/b, by men/women, or by type 1 diabetes (T1D)/type 2 diabetes (T2D) to indicate subpopulations. 


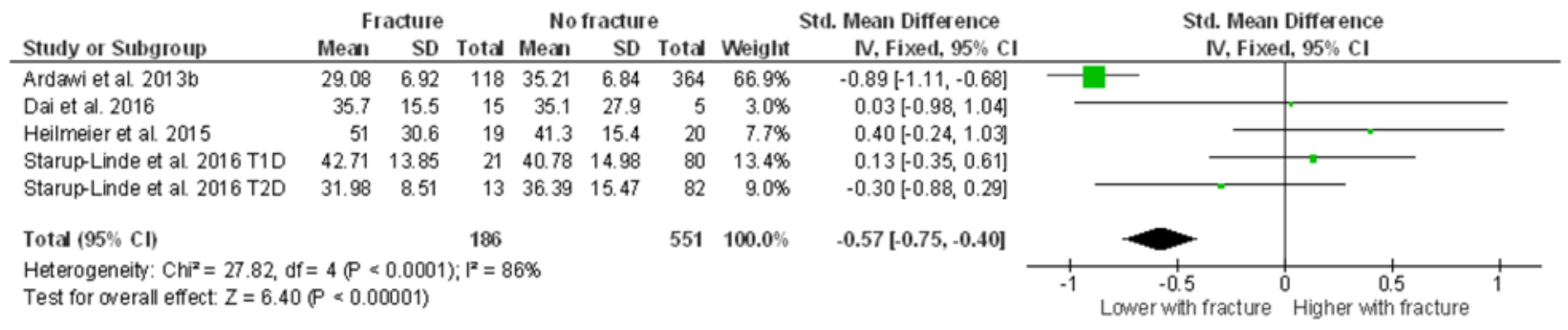

Figure 3 Pooled analysis of procollagen type 1 amino terminal propeptide (P1NP) levels in persons with diabetes with fracture compared with those with diabetes without fracture. Studies with several populations are explained as the author name followed by $a / b$ or by type 1 diabetes (T1D)/type 2 diabetes (T2D) to indicate subpopulations.

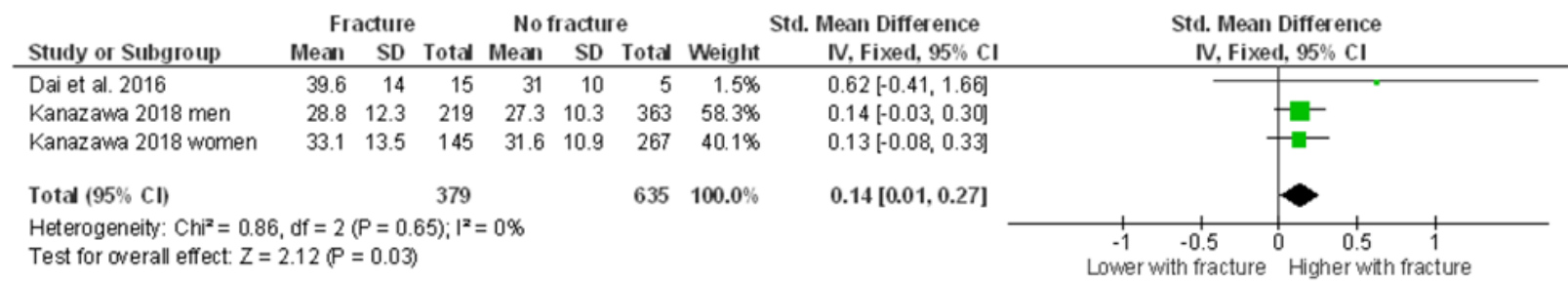

Figure 4 Pooled analysis of bone-specific alkaline phosphatase (BAP) levels in persons with diabetes with fracture compared with those with diabetes without fracture. Studies with several populations are explained as the author name followed by men/women to indicate subpopulations.

\begin{tabular}{|c|c|c|c|c|c|c|c|c|c|c|c|}
\hline \multirow[b]{2}{*}{ Study or Subgroup } & \multicolumn{3}{|c|}{ Fracture } & \multicolumn{3}{|c|}{ No fracture } & \multicolumn{3}{|c|}{ Std. Mean Difference } & \multirow{2}{*}{\multicolumn{2}{|c|}{$\begin{array}{c}\text { Std. Mean Differ ence } \\
\text { IV, Fixed, } 95 \% \mathrm{Cl}\end{array}$}} \\
\hline & Mean & SD & Total & Mean & SD & Total & Weight & IV, Fixed, $95 \% \mathrm{CI}$ & & & \\
\hline Ardawi et al. $2013 b$ & 55.92 & 19.3 & 118 & 44.26 & 13.45 & 364 & $26.5 \%$ & $0.77[0.56,0.98]$ & & & $\longrightarrow$ \\
\hline Dai et al. 2016 & 12.86 & 4.1 & 15 & 10.32 & 33 & 5 & $1.2 \%$ & $0.15[-0.86,1.17]$ & & & \\
\hline Kanazawa 2018 men & 36.5 & 21 & 219 & 38 & 54.3 & 363 & $42.9 \%$ & $-0.03[-0.20,0.13]$ & & & - \\
\hline Kanazawa 2018 women & 55.8 & 40.8 & 145 & 50.2 & 27.7 & 267 & $29.4 \%$ & $0.17[-0.03,0.37]$ & & & \\
\hline Total $(95 \% \mathrm{Cl})$ & & & 497 & & & 999 & $100.0 \%$ & $0.24[0.13,0.35]$ & & & \\
\hline $\begin{array}{l}\text { Heterogeneity: } \mathrm{Chi}^{2}=34 \text {. } \\
\text { Test for overall effect: } Z=\end{array}$ & $\begin{array}{l}6, d f=3 \\
4.32(P\end{array}$ & $\begin{array}{l}\mathbb{P}<0 \\
<0.00\end{array}$ & .00001 & 1); $\left.\right|^{2}=$ & & & & & -1 & -0.5 with fracture & Higher with fracture \\
\hline
\end{tabular}

Figure 5 Pooled analysis of $\mathrm{N}$-terminal cross-linked telopeptide of type 1 collagen (NTX) levels in persons with diabetes with fracture compared with those with diabetes without fracture. Studies with several populations are explained as the author name followed by $\mathrm{a} / \mathrm{b}$ or by men/women to indicate subpopulations. 
(a)

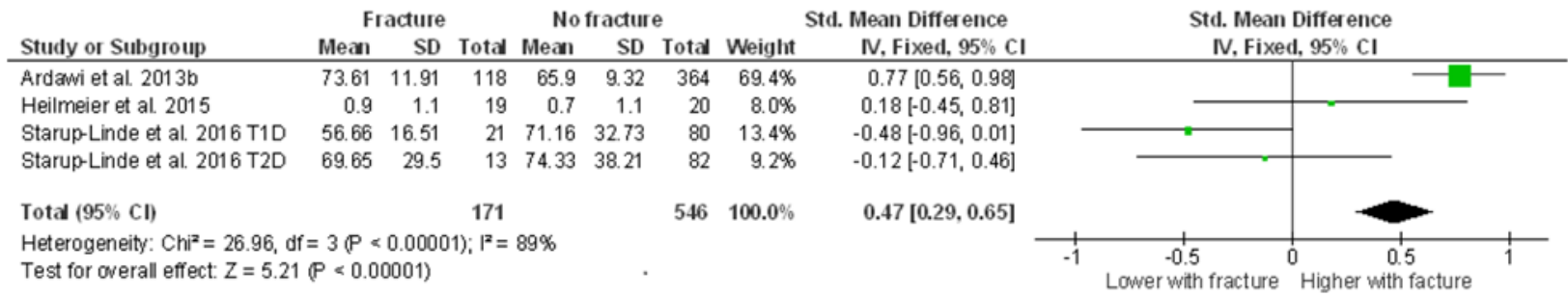

(b)

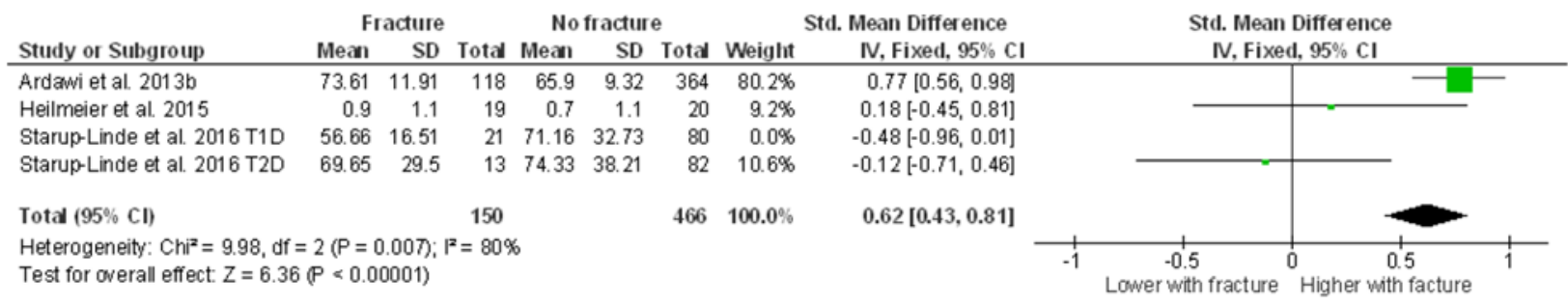

Figure 6 Pooled analysis of sclerostin levels in all persons with diabetes (a) or only participants with type 2 diabetes (T2D) (b) with fracture compared with persons with diabetes without fracture. Studies with several populations are explained as the author name followed by $a / b$, by men/women, or by type 1 diabetes (T1D)/T2D to indicate subpopulations.

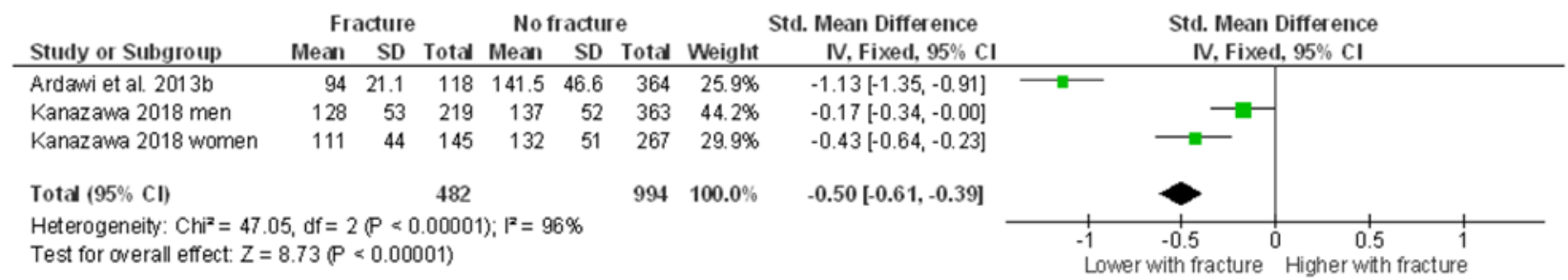

Figure 7 Pooled analysis of insulin-like growth factor-1 (IGF-1) levels in persons with diabetes with fracture compared with persons with diabetes without fracture. Studies with several populations are explained as the author name followed by $a / b$ or by men/women to indicate subpopulations. 


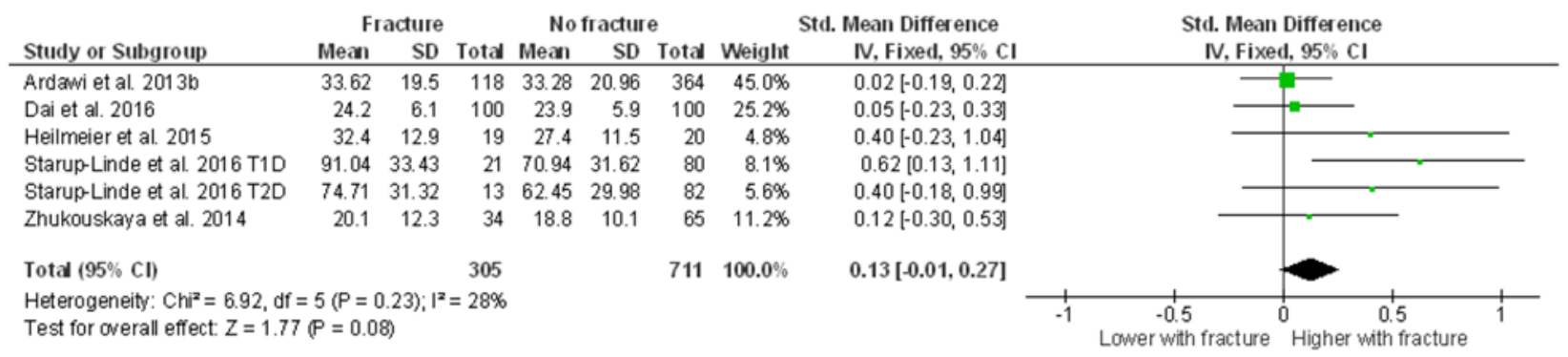

Figure 8 Pooled analysis of Vitamin D levels in persons with diabetes with fracture compared with those with diabetes without fracture. Studies with several populations are explained as the author name followed by a/b or by type 1 diabetes (T1D)/type 2 diabetes (T2D) to indicate subpopulations.

\subsection{Results of Bone Turnover Markers in Secondary Analyses: The Random Effects Model}

Levels of the bone mineralization marker BAP were significantly higher in patients with diabetes with fractures as compared with those without fracture (SMD $=0.14$ [95\% Cl: 0.01, 0.27]; Figure 9). Levels of IGF-1 were significantly lower in patients with diabetes and fracture as compared with those without fracture (SMD $=-0.58$ [95\% $\mathrm{Cl}:-1.13,-0.03]$; Figure 10). For BAP and IGF-1, the use of the MD did not change the results nor the significance of the results. The levels of vitamin $D$ were borderline higher in patients with fracture than in those without fracture $(S M D=0.17$ [95\% Cl: -0.01 . 0.35]; Figure 11); however, results turned non-significant when the MD was used. For all other investigated variables, including osteocalcin, P1NP, CTX, NTX, sclerostin, PTH, and HbA1c, there were no significant differences between the groups in the random effects model in neither the SMD nor the MD (data not shown).

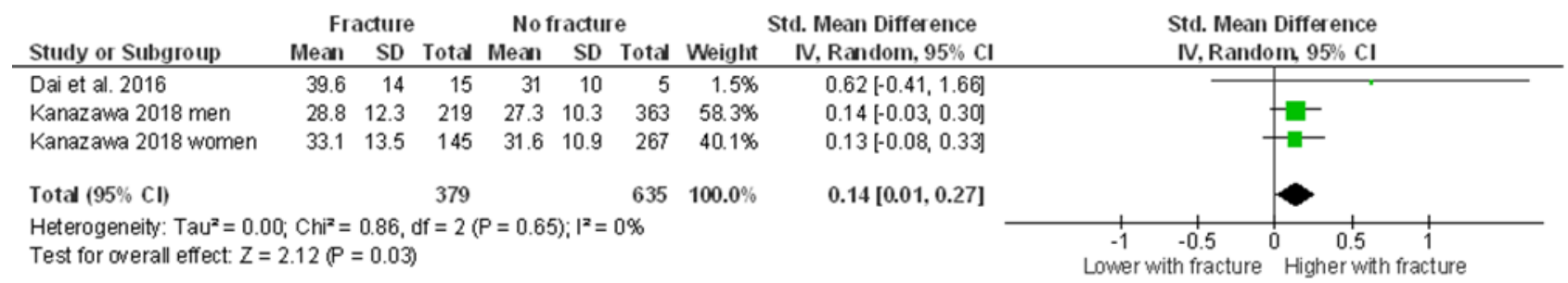

Figure 9 Pooled analysis of bone-specific alkaline phosphatase (BAP) levels in persons with diabetes with fracture compared with persons with diabetes without fracture. Studies with several populations are explained as the author name followed by men/women to indicate subpopulations. 
OBM Geriatrics 2019; 3(4), doi:10.21926/obm.geriatr.1904090

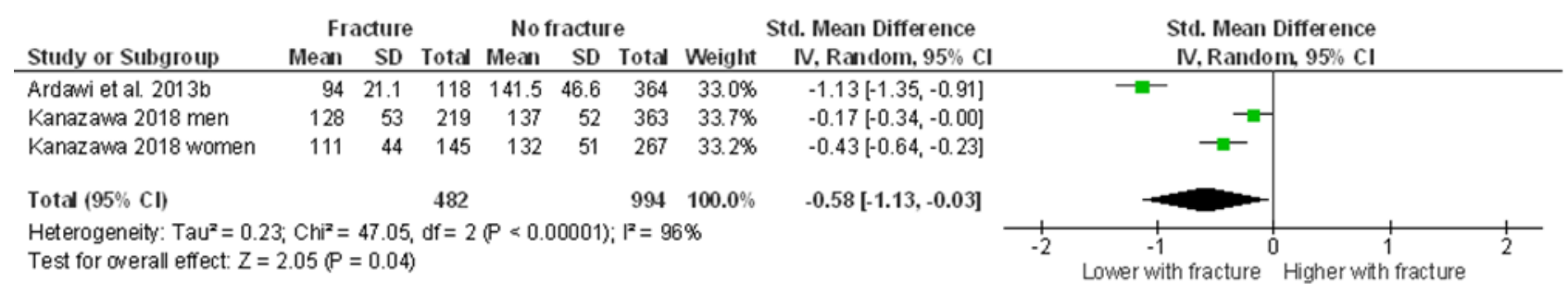

Figure 10 Pooled analysis of insulin-like growth factor-1 (IGF-1) levels in persons with diabetes with fracture compared with those with diabetes without fracture. Studies with several populations are explained as the author name followed by $a / b$ or by men/women to indicate subpopulations.

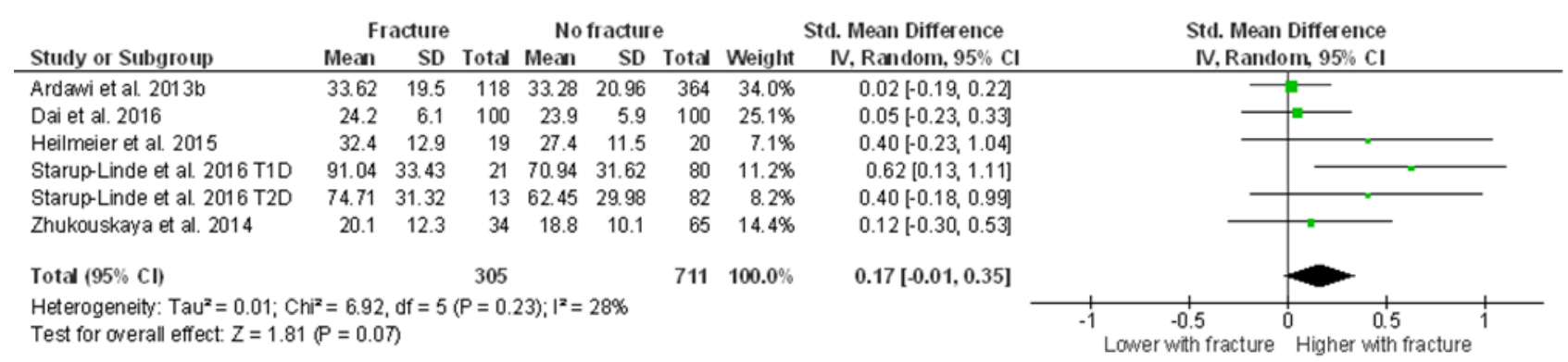

Figure 11 Pooled analysis of Vitamin D levels in persons with diabetes with fracture compared with persons with diabetes without fracture. Studies with several populations are explained as the author name followed by $a / b$, by men/women, or by type 1 diabetes (T1D)/type 2 diabetes (T2D) to indicate subpopulations.

\subsection{Sensitivity Analysis}

First, we wanted to investigate the impact of one large study by Ardawi et al. [35]. We suspected that the study was the main determinant of the pooled estimates for osteocalcin (Figure 2), P1NP (Figure 3), NTX (Figure 5), sclerostin (Figure 6), and IGF-1 (Figure 7). Hence, we repeated all analyses using the fixed effects model with the omission of the study by Ardawi et al. (data not shown). For bone resorption markers, the levels of NTX were found to be nonsignificantly different in patients with fracture as compared with those without fracture (SMD = 0.05 [95\% Cl: $-0.08,0.18]$ ); however and interestingly, the levels of CTX changed from nonsignificantly different to significantly lower in patients with fracture (SMD $=-0.30[95 \% \mathrm{Cl}:-0.52,-$ 0.08]). For formation markers, osteocalcin and P1NP, and also for sclerostin, results were nonsignificant in the sensitivity analysis. For IGF-1, sensitivity analysis was not possible owing to a small number of studies. The levels of vitamin D turned significantly higher (SMD $=0.22[95 \% \mathrm{Cl}$ : $0.03,0.40])$ in patients with fracture compared with those without fracture. The levels of PTH and $\mathrm{HbA1c}$ remained non-significantly different between the groups.

Second, we performed sensitivity analyses to explore the effect of study design and therefore performed all analyses using the fixed effects model while excluding the only prospective study by Dai et al. [36]. In brief, levels of OC and P1NP remained lower in patients with fracture as compared with those without fracture, $(\mathrm{SMD}=-0.37[95 \% \mathrm{Cl}:-0.47,-0.27])$ and $(\mathrm{SMD}=-0.59$ $(95 \% \mathrm{Cl}:-0.77,-0.41])$, respectively. The levels of NTX remained higher in patients with fracture as 
compared with those without fracture (SMD $=0.24[95 \% \mathrm{Cl}: 0.13,0.35])$, whereas results for CTX remained non-significant (SMD $=-0.10[95 \% \mathrm{Cl}:-0.25,0.05])$.

\section{Discussion}

In the present meta-analysis of observational studies, we found that patients with diabetes with fracture had higher levels of BAP and lower levels of IGF-1 as compared with those with diabetes and without fracture. However, the results differed in primary and secondary analyses when applying either the fixed or the random effects model, respectively. In the primary analyses using the fixed effects model, patients with diabetes and fracture reported lower levels of formative BTMs and IGF-1 and higher levels of bone resorption marker NTX and sclerostin as compared with those with diabetes and without fracture. Thus, bone turnover appeared to be associated with fractures in diabetes; lower bone formation was possibly caused by higher levels of sclerostin and lower levels of IGF-1. However, interpretation of these results is limited by the effect of one large study, as shown in sensitivity analyses where results for osteocalcin, P1NP, NTX, and sclerostin became non-significant. In secondary analyses using the random-effects model, only levels of BAP and IGF-1 were significantly different depending on the fracture status.

In a previous meta-analysis, we demonstrated diabetes to be a state of low levels of BTMs, possibly owing to multiple factors, including increased levels of sclerostin [10]. The present study suggests an association between the levels of bone formation markers and fracture status in diabetes. This is an important finding that warrants further investigation in the form of longitudinal studies as the risk of fracture is underestimated in patients with diabetes when applying traditional osteoporosis risk factors [14].

Our results indicate that the presence of low levels of IGF-1 and high levels of sclerostin in patients with diabetes with fracture could reflect an osteocyte dysfunction. Osteocytes produce sclerostin, a potent inhibitor of the Wnt signaling pathway and a known suppressor of bone formation via inhibition of osteoblast formation and activity [39]. Currently, a monoclonal antibody inhibiting sclerostin is under development to treat osteoporosis ("Romosozumab") [40]. Romosozumab has been approved by the Food and Drug Administration and it will be interesting to observe the effect of this drug on bone turnover and fracture risk in patients with T1D and T2D. However, based on the findings of the present meta-analysis, we would expect a prominent effect.

IGF-1 has a modulating effect on bone. Circulating IGF-1 is mainly secreted from the liver and produced locally in osteocytes to a lesser extent. It inhibits osteoblast apoptosis and increases osteoblastogenesis [41, 42]. IGF-1 production is regulated by growth hormone and diet. IGF-1 produced locally in osteocytes mediates mechanosensitivity in bone [43] and promotes bone formation [44]. The circulating IGF-1 measured in the studies included in the meta-analysis reflects hepatic and osteocyte production. Both sources of IGF-I have been shown to stimulate longitudinal bone growth and are thus bone anabolic [45]. However, studies in animals have reported that liver-derived IGF-1 contributes only marginally to bone growth and regulation [46]. Therefore, the magnitude of the effect of hepatic IGF-1 on bone formation is controversial. Mice with osteocyte-specific knockout of IGF-1 have impaired bone growth and decreased bone turnover with lower levels of P1NP and CTX [47]. Diabetes has been associated with low IGF-1levels [48] although the results are conflicting [49]. Based on our results, we speculate whether unknown factors in diabetes promote changes in osteocyte function. The osteocyte dysfunction 
may facilitate changes in the levels of IGF-1 and sclerostin that affect the surrounding bone cells, thus changing the bone turnover and fracture risk (Figure 12). However, currently available data are based on observational data from cross-sectional studies that do not allow to reach a conclusion concerning the cause and effect.

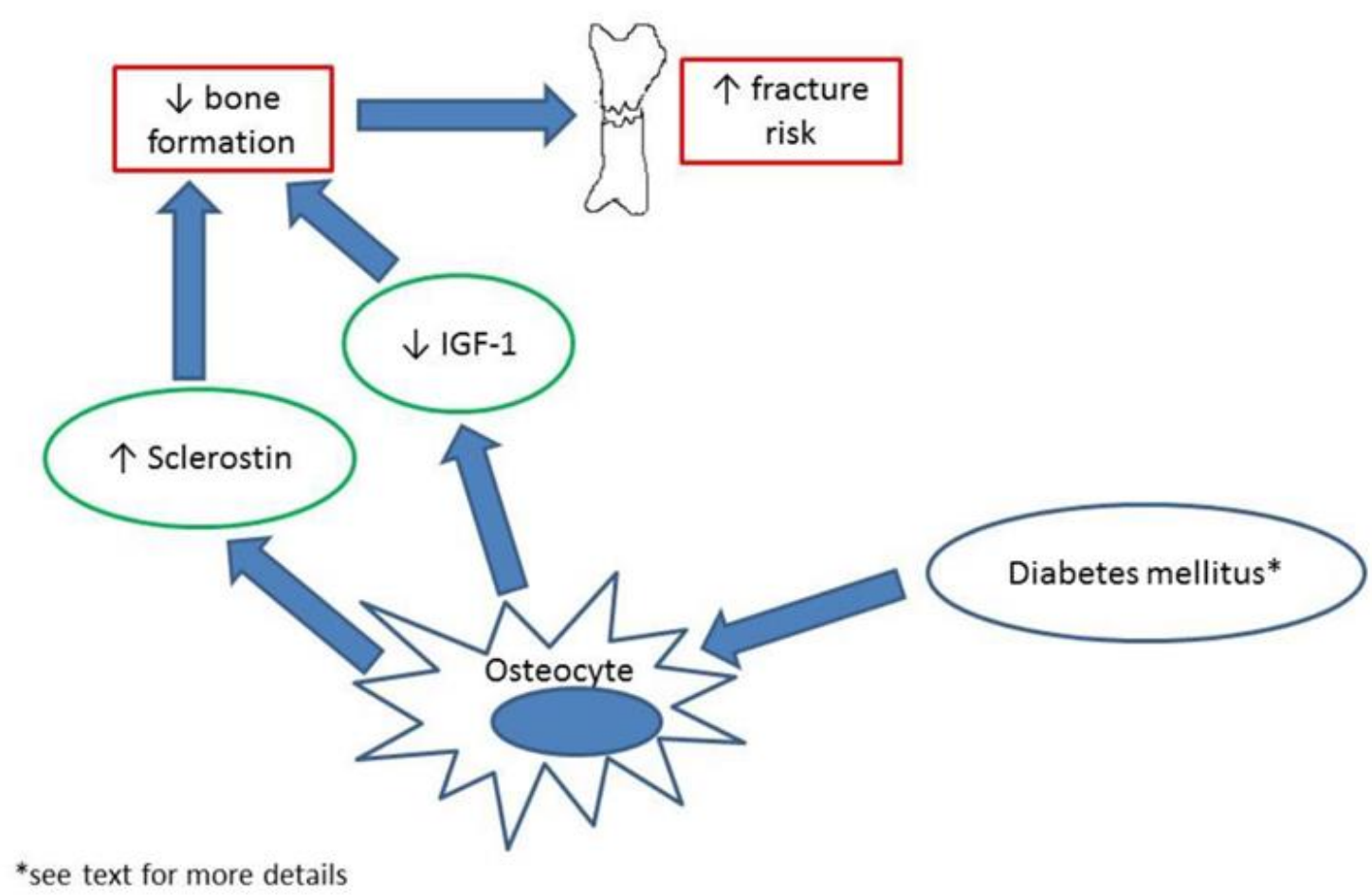

Figure 12 Overview of the proposed mechanisms that promote changes in osteocyte function leading to increased fracture risk for some persons with diabetes. Multiple factors in diabetes including hyperglycemia, a relatively sedentary lifestyle, and microvascular changes may promote osteocyte dysfunction. Osteocyte dysfunction may, in turn, lead to increased sclerostin levels, decreased IGF-1 levels and hence decreased bone formation that ultimately results in increased fracture risk for the subgroup of persons with diabetes.

In a previous meta-analysis of observational studies, increased levels of P1NP and CTX were associated with increased fracture risk in the general population [16]. The results of the present analysis are in contrast with this, as we found an association between low levels of P1NP and fracture status in diabetes. This observation might suggest that different fracture mechanisms may be involved in patients with diabetes and without diabetes. Furthermore, although NTX levels were higher in patients with diabetes with fracture, we did not find any significant differences in the levels of the resorptive marker CTX in patients with or without fracture in both analyses. Whether this was attributed to insufficient statistical power or because only bone formation and not bone resorption differed between patients with diabetes with and without fracture, is a limitation to the interpretation of the results. We observed higher levels of BAP in patients with fracture as compared with those without fracture, suggesting increased mineralization in patients with fracture, despite apparently decreased bone formation. However, the increased levels of BAP in the fracture group could be influenced by fracture repair mechanisms. Most studies did not 
provide any information on the time from fracture incidence to analyses of levels of BMTs; however, levels of BMTs are reported to change rapidly in the time following hip [50] and vertebral [51] fractures in patients without diabetes. Recent fractures in some of the participants in the group of fracture patients may, therefore, have resulted in a higher variation in levels of BTMs.

We primarily applied the fixed effects model in our analyses. The fixed effects model assumes that all included studies report on the same effect size; therefore, any inconsistency between the studies is solely attributed to random sampling error. An advantage of using the fixed effects model is that the included studies appear to be relatively similar concerning study population (inclusion and exclusion criteria) and effect measure (fractures). A limitation of the fixed effects model is that larger studies weigh more than smaller studies in the pooled estimate. Therefore, we conducted a sensitivity analysis, where the largest study was excluded. In contrary to the fixed effects model, the random effects model, which we used in secondary analyses, assumes that the effect size is different in the included studies owing to heterogeneity or different study characteristics. Consequently, the random effects model weighs large and small studies accordingly. There is no definitive conclusion when selecting the appropriate model for analysis.

In the sensitivity analysis using the fixed effects model, the effect of study size was investigated by excluding the study by Ardawi et al. [35]. Consequently, the levels of formative BTMs were not significantly different between patients with diabetes with and without fracture. Furthermore, the difference in the levels of NTX was non-significant between the groups, whereas the levels of CTX changed to significantly lower in patients with diabetes and fracture as compared with those without fracture. In the study by Ardawi et al., exclusion criteria were similar to the other studies; however, a higher number of cases without fracture were included, and most notably, participants had higher levels of $\mathrm{HbA} 1 \mathrm{c}$ and a higher BMI. As both the non-fracture and the fracture groups had higher $\mathrm{HbA} 1 \mathrm{c}$ and $\mathrm{BMI}$, selection bias was unlikely. Taken together, results from the sensitivity analyses showed that a single study was responsible for a large part of the pooled risk estimate, and this limited the interpretation of the results. However, the study design did not appear to affect the results when sensitivity analyses were performed by excluding the only study with a prospective design by Dai et al. [36].

The strengths of the present study are the thorough study selection and the overall high study quality. All studies, except for one, excluded participants with renal failure, which is known to affect bone turnover and BTMs. For the remaining study, we expect that any renal disease would be present in both the compared groups and would balance out any possible confounding effect. To assess any possible inter-assay variation, the type of assay employed in the analyses of levels of BTMs were screened. For instance, for sclerostin, an assay from Biomedica was used in all studies except for the study by Heilmeier et al. [37]. Concerning P1NP, a number of different assays were employed. All pooled estimates were reported as SMD, as this took into account both inter-assay and intra-laboratory variations.

A limitation to our study was that the included studies investigated a wide range of fractures with some studies only including vertebral fractures and others including fragility fractures or all fractures including possible traumatic fractures. This diversity in the fracture site makes the results valid to fractures in general in patients with diabetes; however, it may, in turn, limit the power of the study. The included studies were from Asia, Europe, North America, and The Middle East. It is well-known that the prevalence of insulin resistance and osteoporosis in the eastern and the 
western parts of the world differs; however, it is beyond the scope of this review to speculate whether the mechanism of fracture in patients with diabetes may also diverge in different ethnic populations. However, in each study, the case and the control groups had comparable ethnicity.

Another limitation to our study was the observational and cross-sectional design of the included studies, preventing us to conclude on causality. Owing to the cross-sectional nature of the studies, it was not possible to address whether low levels of certain BTMs caused fractures or vice versa.

Two of the studies [30,36] collected blood samples in a non-fasting state, which is known to affect measurements of CTX [52]. Furthermore, not all studies excluded patients with high fall risk or adjusted for falls in the data analysis. Fall risk may predispose some patients with diabetes to an increased risk of fracture and we could not exclude the possibility that patients with fracture had an increased fall risk owing to more severe disease and occurrence of diabetes complications. Anti-diabetic drugs may influence bone turnover in different ways. However, all included studies provided limited information on the use of anti-diabetic medication. This prevented the possibility of adjusting for plausible increased fracture risks as a side-effect of anti-diabetic drugs, such as thiazolidinediones, which were previously widely used in the treatment of diabetes; however, these are now known to increase the risk of fracture [53]. Most studies excluded participants taking anti-osteoporotic medications, an important confounder, as participants with previous fractures could be susceptible to anti-osteoporotic treatment that could, in turn, alter the bone turnover and levels of circulating BTMs. For vitamin D, two studies included participants taking supplementation. One study reported no difference in circulating vitamin $D$ levels between the fracture and the no-fracture groups [38], whereas the other study found a positive correlation between vitamin D levels and previous major osteoporotic fracture in sensitivity analyses [30]. Not all studies provided information on the duration of diabetes. Most of our analyses employed both patients with T1D and T2D, except for sclerostin where the analyses were performed with and without patients with T1D. Changes in the bone turnover are potentially triggered by different pathophysiological mechanisms in T1D and T2D, which potentially confound the estimates.

\section{Conclusions}

In conclusion, we observed that individuals with diabetes and fracture had higher levels of BAP and lower levels of IGF-1 as compared with those without fractures. Furthermore, the data suggested that patients with diabetes and fracture had lower levels of bone formation markers OC and P1NP and also IGF-1, and higher levels of the bone resorption marker NTX and also sclerostin as compared with those without fracture. However, some results were dependent on one large study, and hence, we could only speculate an association between reduced bone formation in patients with diabetes and fracture status.

The cross-sectional design of studies included in our analyses did not allow us to provide a conclusion on causality. To evaluate whether levels of BTMs could predict the risk of fracture in diabetes, further longitudinal studies are warranted, especially to investigate changes in BTMs over time in patients with diabetes and occurrence of fracture.

\section{Acknowledgments}

None. 


\section{Author Contributions}

$\mathrm{KH}$ and JS-L initially conceived the idea for the study and performed the literature searches and screening of papers. All authors were involved in the statistical analyses, the interpreting of data, and the writing of the paper. All authors approved the final version of the paper before publication.

\section{Funding}

None.

\section{Competing Interests}

BL reports research grants (institution) from Amgen and Novo Nordisk. BL serves on advisory boards for Amgen, Eli Lilly, and UCB and has received honoraria for lecturing from Amgen, Eli Lilly, UCB and TEVA. TH has received lecture fees from Eli Lilly, Amgen, and Astra-Zeneca. JS-L reports personal fees from Gilead Sciences Denmark and personal fees from Eli Lilly Denmark, outside the submitted work.

\section{References}

1. Janghorbani M, Van Dam RM, Willett WC, Hu FB. Systematic review of type 1 and type 2 diabetes mellitus and risk of fracture. Am J Epidemiol. 2007; 166: 495-505.

2. Liao CC, Lin CS, Shih CC, Yeh CC, Chang YC, Lee YW, et al. Increased risk of fracture and postfracture adverse events in patients with diabetes: Two nationwide population-based retrospective cohort studies. Diabetes Care. 2014; 37: 2246-2252.

3. Gulcelik NE, Bayraktar M, Caglar O, Alpaslan M, Karakaya J. Mortality after hip fracture in diabetic patients. Exp Clin Endocrinol Diabetes. 2011; 119: 414-418.

4. Napoli N, Strotmeyer ES, Ensrud KE, Sellmeyer DE, Bauer DC, Hoffman AR, et al. Fracture risk in diabetic elderly men: The MrOS study. Diabetologia. 2014; 57: 2057-2065.

5. Yamamoto M, Sugimoto T. Advanced Glycation End Products, Diabetes, and Bone Strength. Curr Osteoporos Rep. 2016; 14: 320-326.

6. Gennari L, Merlotti D, Valenti R, Ceccarelli E, Ruvio M, Pietrini MG, et al. Circulating sclerostin levels and bone turnover in type 1 and type 2 diabetes. J Clin Endocrinol Metab. 2012; 97: 1737-1744.

7. Starup-Linde J, Lykkeboe S, Gregersen S, Hauge EM, Langdahl BL, Handberg A, et al. Differences in biochemical bone markers by diabetes type and the impact of glucose. Bone. 2016; 83: 149-155.

8. Palermo A, D'Onofrio L, Eastell R, Schwartz AV, Pozzilli P, Napoli N. Oral anti-diabetic drugs and fracture risk, cut to the bone: Safe or dangerous? A narrative review. Osteoporos Int. 2015; 26: 2073-2089.

9. Napoli N, Chandran M, Pierroz DD, Abrahamsen B, Schwartz AV, Ferrari SL, et al. Mechanisms of diabetes mellitus-induced bone fragility. Nat Rev Endocrinol. 2017; 13: 208-219. 
10. Hygum K, Starup-Linde J, Harslof T, Vestergaard P, Langdahl BL. Mechanisms in endocrinology: Diabetes mellitus, a state of low bone turnover - a systematic review and meta-analysis. Eur J Endocrinol. 2017; 176: R137-R157.

11. Marshall $D$, Johnell $O$, Wedel $H$. Meta-analysis of how well measures of bone mineral density predict occurrence of osteoporotic fractures. BMJ. 1996; 312: 1254-1259.

12. Kanis JA, Oden A, Johansson H, Borgstrom F, Strom O, McCloskey E. FRAX and its applications to clinical practice. Bone. 2009; 44: 734-743.

13. Vestergaard P. Discrepancies in bone mineral density and fracture risk in patients with type 1 and type 2 diabetes--a meta-analysis. Osteoporos Int. 2007; 18: 427-444.

14. Giangregorio LM, Leslie WD, Lix LM, Johansson H, Oden A, McCloskey E, et al. FRAX underestimates fracture risk in patients with diabetes. J Bone Miner Res. 2012; 27: 301-308.

15. Vasikaran S, Eastell R, Bruyere O, Foldes AJ, Garnero P, Griesmacher A, et al. Markers of bone turnover for the prediction of fracture risk and monitoring of osteoporosis treatment: A need for international reference standards. Osteoporos Int. 2011; 22: 391-420.

16. Johansson H, Oden A, Kanis JA, McCloskey EV, Morris HA, Cooper C, et al. A meta-analysis of reference markers of bone turnover for prediction of fracture. Calcif Tissue Int. 2014; 94: 560567.

17. Liberati A, Altman DG, Tetzlaff J, Mulrow C, Gotzsche PC, loannidis JP, et al. The PRISMA statement for reporting systematic reviews and meta-analyses of studies that evaluate healthcare interventions: Explanation and elaboration. BMJ. 2009; 339: b2700.

18. Kanazawa I, Yamaguchi T, Yamamoto M, Yamauchi M, Yano S, Sugimoto T. Serum insulin-like growth factor-I level is associated with the presence of vertebral fractures in postmenopausal women with type 2 diabetes mellitus. Osteoporos Int. 2007; 18: 1675-1681.

19. Kanazawa I, Yamaguchi T, Yamamoto M, Yamauchi M, Yano S, Sugimoto T. Relationships between serum adiponectin levels versus bone mineral density, bone metabolic markers, and vertebral fractures in type 2 diabetes mellitus. Eur J Endocrinol. 2009; 160: 265-273.

20. Kanazawa I, Yano S, Yamaguchi T, Notsu Y, Nabika T, Sugimoto T. Relationships between dimethylarginine and the presence of vertebral fractures in type 2 diabetes mellitus. Clin Endocrinol (Oxf). 2010; 73: 463-468.

21. Kanazawa I, Yamaguchi T, Yamamoto M, Sugimoto T. Relationship between treatments with insulin and oral hypoglycemic agents versus the presence of vertebral fractures in type 2 diabetes mellitus. J Bone Miner Metab. 2010; 28: 554-560.

22. Kanazawa I, Yamaguchi T, Sugimoto T. Serum insulin-like growth factor-I is a marker for assessing the severity of vertebral fractures in postmenopausal women with type 2 diabetes mellitus. Osteoporos Int. 2011; 22: 1191-1198.

23. Notsu M, Kanazawa I, Tanaka S, Yamaguchi T, Sugimoto T. Serum dipeptidyl peptidase-4 is associated with multiple vertebral fractures in type 2 diabetes mellitus. Clin Endocrinol (Oxf). 2016; 84: 332-337.

24. Miyake H, Kanazawa I, Sugimoto T. Decreased serum insulin-like growth factor-I is a risk factor for non-vertebral fractures in diabetic postmenopausal women. Intern Med. 2017; 56: 269-273.

25. Tanaka KI, Kanazawa I, Kaji H, Sugimoto T. Association of osteoglycin and FAM5C with bone turnover markers, bone mineral density, and vertebral fractures in postmenopausal women with type 2 diabetes mellitus. Bone. 2016; 95: 5-10. 
26. Yamamoto $M$, Yamauchi $M$, Sugimoto $T$. Elevated sclerostin levels are associated with vertebral fractures in patients with type 2 diabetes mellitus. J Clin Endocrinol Metab. 2013; 98: 4030-4037.

27. Yamamoto M, Yamaguchi T, Yamauchi M, Yano S, Sugimoto T. Serum pentosidine levels are positively associated with the presence of vertebral fractures in postmenopausal women with type 2 diabetes. J Clin Endocrinol Metab. 2008; 93: 1013-1019.

28. Kanazawa I, Notsu M, Miyake H, Tanaka K, Sugimoto T. Assessment using serum insulin-like growth factor-I and bone mineral density is useful for detecting prevalent vertebral fractures in patients with type 2 diabetes mellitus. Osteoporos Int. 2018; 29: 2527-2535.

29. Kanazawa I, Yamaguchi T, Yamamoto $M$, Yamauchi $M$, Yano S, Sugimoto T. Serum osteocalcin/bone-specific alkaline phosphatase ratio is a predictor for the presence of vertebral fractures in men with type 2 diabetes. Calcif Tissue Int. 2009; 85: 228-234.

30. Starup-Linde J, Lykkeboe S, Gregersen S, Hauge EM, Langdahl BL, Handberg A, et al. Bone structure and predictors of fracture in type 1 and type 2 diabetes. J Clin Endocrinol Metab. 2016; 101: 928-936.

31. Herzog R, Alvarez-Pasquin MJ, Diaz C, Del Barrio JL, Estrada JM, Gil A. Are healthcare workers' intentions to vaccinate related to their knowledge, beliefs and attitudes? A systematic review. BMC Public Health. 2013; 13: 154.

32. Oei L, Zillikens MC, Dehghan A, Buitendijk GH, Castano-Betancourt MC, Estrada K, et al. High bone mineral density and fracture risk in type 2 diabetes as skeletal complications of inadequate glucose control: The Rotterdam Study. Diabetes Care. 2013; 36: 1619-1628.

33. Puar TH, Khoo JJ, Cho LW, Xu Y, Chen YT, Chuo AM, et al. Association between glycemic control and hip fracture. J Am Geriatr Soc. 2012; 60: 1493-1497.

34. Neumann $T$, Lodes $S$, Kästner $B$, Franke $S$, Kiehntopf $M$, Lehmann $T$, et al. High serum pentosidine but not esRAGE is associated with prevalent fractures in type 1 diabetes independent of bone mineral density and glycaemic control. Osteoporos Int. 2014; 25: 15271533.

35. Ardawi MS, Akhbar DH, Alshaikh A, Ahmed MM, Qari MH, Rouzi AA, et al. Increased serum sclerostin and decreased serum IGF-1 are associated with vertebral fractures among postmenopausal women with type-2 diabetes. Bone. 2013; 56: 355-362.

36. Dai Z, Wang R, Ang LW, Yuan JM, Koh WP. Bone turnover biomarkers and risk of osteoporotic hip fracture in an Asian population. Bone. 2016; 83: 171-177.

37. Heilmeier U, Carpenter DR, Patsch JM, Harnish R, Joseph GB, Burghardt AJ, et al. Volumetric femoral BMD, bone geometry, and serum sclerostin levels differ between type 2 diabetic postmenopausal women with and without fragility fractures. Osteoporos Int. 2015; 26: 12831293.

38. Zhukouskaya VV, Eller-Vainicher C, Gaudio A, Cairoli E, Ulivieri FM, Palmieri S, et al. In postmenopausal female subjects with type 2 diabetes mellitus, vertebral fractures are independently associated with cortisol secretion and sensitivity. J Clin Endocrinol Metab. 2015; 100: 1417-1425.

39. Baron R, Kneissel M. WNT signaling in bone homeostasis and disease: From human mutations to treatments. Nat Med. 2013; 19: 179-192.

40. Cosman F, Crittenden DB, Adachi JD, Binkley N, Czerwinski E, Ferrari S, et al. Romosozumab treatment in postmenopausal women with osteoporosis. N Engl J Med. 2016; 375: 1532-1543. 
41. Johansson $A G$, Lindh $E$, Ljunghall $S$. Insulin-like growth factor I stimulates bone turnover in osteoporosis. Lancet. 1992; 339: 1619.

42. Locatelli V, Bianchi VE. Effect of GH/IGF-1 on bone metabolism and osteoporsosis. Int J Endocrinol. 2014; 2014: 235060.

43. Sheng $\mathrm{MH}, \mathrm{Lau} \mathrm{KH}$, Baylink DJ. Role of osteocyte-derived insulin-like growth factor $\mathrm{i}$ in developmental growth, modeling, remodeling, and regeneration of the bone. J Bone Metab. 2014; 21: 41-54.

44. Behringer RR, Lewin TM, Quaife CJ, Palmiter RD, Brinster RL, D'Ercole AJ. Expression of insulin-like growth factor I stimulates normal somatic growth in growth hormone-deficient transgenic mice. Endocrinology. 1990; 127: 1033-1040.

45. Ohlsson C, Mohan S, Sjogren K, Tivesten A, Isgaard J, Isaksson O, et al. The role of liverderived insulin-like growth factor-I. Endocr Rev. 2009; 30: 494-535.

46. Sjogren K, Liu JL, Blad K, Skrtic S, Vidal O, Wallenius V, et al. Liver-derived insulin-like growth factor I (IGF-I) is the principal source of IGF-I in blood but is not required for postnatal body growth in mice. Proc Natl Acad Sci U S A. 1999; 96: 7088-7092.

47. Sheng $M H$, Zhou XD, Bonewald LF, Baylink DJ, Lau KH. Disruption of the insulin-like growth factor-1 gene in osteocytes impairs developmental bone growth in mice. Bone. 2013; 52: 133144.

48. Teppala S, Shankar A. Erratum. Association between serum IGF-1 and diabetes among U.S. Adults. Diabetes Care 2010;33:2257-2259. Diabetes Care. 2017; 40: 1133.

49. Clemmons DR. Metabolic actions of insulin-like growth factor-I in normal physiology and diabetes. Endocrinol Metab Clin North Am. 2012; 41: 425-443, vii-viii.

50. Yu-Yahiro JA, Michael RH, Dubin NH, Fox KM, Sachs M, Hawkes WG, et al. Serum and urine markers of bone metabolism during the year after hip fracture. J Am Geriatr Soc. 2001; 49: 877-883.

51. Pan C, Liu X, Li T, Wang G, Sun J. Kinetic of bone turnover markers after osteoporotic vertebral compression fractures in postmenopausal female. J Orthop Surg Res. 2018; 13: 314.

52. Bjarnason NH, Henriksen EE, Alexandersen P, Christgau S, Henriksen DB, Christiansen C. Mechanism of circadian variation in bone resorption. Bone. 2002; 30: 307-313.

53. Meier C, Schwartz AV, Egger A, Lecka-Czernik B. Effects of diabetes drugs on the skeleton. Bone. 2016; 82: 93-100. 


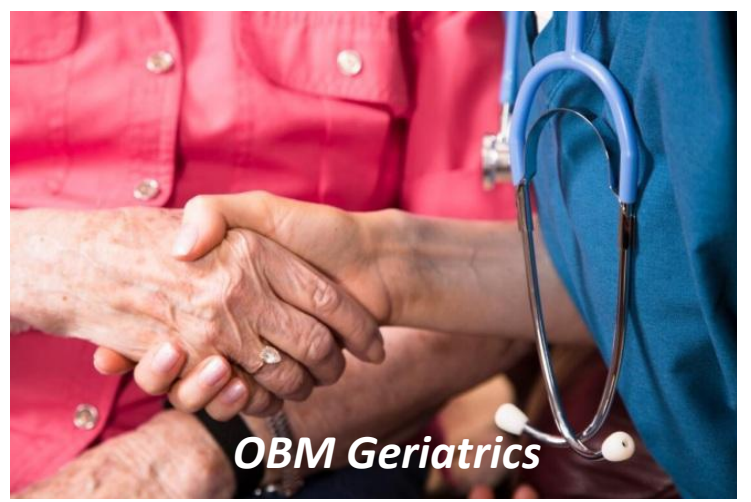

Enjoy $O B M$ Geriatrics by:

1. Submitting a manuscript

2. Joining in volunteer reviewer bank

3. Joining Editorial Board

4. Guest editing a special issue

For more details, please visit: http://www.lidsen.com/journals/geriatrics 